\title{
Identification of a low-molecular weight TrkB antagonist with anxiolytic and antidepressant activity in mice
}

\author{
Maxime Cazorla, ${ }^{1,2}$ Joël Prémont, ${ }^{1}$ Andre Mann, ${ }^{3}$ Nicolas Girard, ${ }^{3}$ \\ Christoph Kellendonk, ${ }^{2}$ and Didier Rognan ${ }^{3}$
}

\begin{abstract}
${ }^{1}$ Neurobiology and Molecular Pharmacology, Centre de Psychiatrie et Neurosciences, UMR-894 INSERM/Université Paris Descartes, Paris, France. 2Department of Pharmacology and Psychiatry, Columbia University, New York, New York, USA. ${ }^{3}$ Structural Chemogenomics, Laboratory of Therapeutic Innovation, UMR-7200 CNRS/Université de Strasbourg, Illkirch, France.
\end{abstract}

\begin{abstract}
The neurotrophin brain-derived neurotrophic factor (BDNF) and its receptor tropomyosin-related kinase $B$ (TrkB) have emerged as key mediators in the pathophysiology of several mood disorders, including anxiety and depression. However, therapeutic compounds that interact with TrkB receptors have been difficult to develop. Using a combination of structure-based in silico screening and high-capacity functional assays in recombinant and neuronal cells, we identified a low-molecular weight TrkB ligand (ANA-12) that prevented activation of the receptor by BDNF with a high potency. ANA-12 showed direct and selective binding to TrkB and inhibited processes downstream of TrkB without altering TrkA and TrkC functions. KIRA-ELISA analysis demonstrated that systemic administration of ANA-12 to adult mice decreased TrkB activity in the brain without affecting neuronal survival. Mice administered ANA-12 demonstrated reduced anxiety- and depressionrelated behaviors on a variety of tests predictive of anxiolytic and antidepressant properties in humans. This study demonstrates that structure-based virtual screening strategy can be an efficient method for discovering potent TrkB-selective ligands that are active in vivo. We further propose that ANA-12 may be a valuable tool for studying BDNF/TrkB signaling and may constitute a lead compound for developing the next generation of therapeutic agents for the treatment of mood disorders.
\end{abstract}

\section{Introduction}

Neurotrophins are a family of growth factor hormones that regulate development and maintenance of the central and peripheral nervous systems by interacting with the tropomyosin-related kinase (Trk) receptors. Nerve growth factor (NGF) binds preferentially to TrkA, brain-derived neurotrophic factor (BDNF) and neurotrophin-4/5 (NT-4/5) bind to TrkB, and neurotrophin-3 (NT-3) binds to TrkC. All neurotrophins also interact with the nonselective $\mathrm{p} 75^{\mathrm{NTR}}$ receptor with similar affinities (1). Interaction of BDNF with TrkB triggers receptor dimerization, transphosphorylation of intracellular tyrosine residues, and subsequent activation of the 3 major signaling pathways involving MAPK, PI3K, and phospholipase C- $\gamma(2)$.

Given its trophic effect on neurons and its contribution to the regulation of synaptic transmission and plasticity $(3,4)$, the BDNF/ TrkB system has been proposed as a key element in the pathophysiology of several mood disorders, including drug addiction, depression, and anxiety (5-7). Accordingly, BDNF signaling in the stress system of the brain, including the hippocampus and the hypothalamo-pituitary-adrenocortical (HPA) axis, is thought to be a potential target for antidepressant action (8-10). Positive modulators of BDNF/TrkB signaling have therefore been proposed to have antidepressive potential in humans (11). However, recent studies have challenged this hypothesis, and the role of BDNF in the pathogenesis of depression and anxiety is still controversial.

In contrast to its function on antidepressant efficacy in the hippocampus and HPA axis, BDNF induces long-term neuronal

Conflict of interest: The authors have declared that no conflict of interest exists. Citation for this article: J Clin Invest. 2011;121(5):1846-1857. doi:10.1172/JCI43992. changes in the reward circuitry of rodents that predict the facilitation of depression and stress-induced anxiety in humans $(7,12)$. In these studies, preventing BDNF production in dopaminergic regions or inhibiting BDNF/TrkB signaling in the nucleus accumbens reduced the anxiety toward former aggressors and induces strong antidepressive effects in rodent, predicting that TrkB inhibition may lead to antidepressant action in humans $(7,12)$. In line with this observation, mice susceptible to stress-related anxiety and depression exhibit high levels of BDNF in the ventral striatum (13) and acutely stressed mice show a rapid and sustained increase in BDNF expression in the prefrontal cortex and in the hippocampus $(14,15)$. In consequence, while inhibiting BDNF signaling may not lead to depression-like behaviors (16-18), reports have suggested that inhibiting TrkB signaling may in contrast be a therapeutic strategy for treating human mood disorders $(5,7,13,19)$.

Despite their potential clinical significance, small-molecule antagonists of TrkB are not available. The development of lowmolecular weight compounds ( $<500 \mathrm{Da})$ that can selectively antagonize a large $145-\mathrm{kDa}$ dimeric TrkB complex activated by a $27-\mathrm{kDa}$ dimeric ligand has been difficult to address. We recently developed a highly potent small peptide that allosterically inhibits TrkB signaling in neurons and that is active in vivo after systemic delivery (20). Our in vivo study, together with others' studies (21-23), demonstrates the feasibility of developing heterocyclic ligands that can modulate TrkB activity in living animals. However, peptide-derived ligands have the problem of being proteolytically unstable, which potentially decreases their efficiency under therapeutic conditions. Here, we aimed at identifying small, proteolytically stable modulators that are selective for TrkB and active in vivo. To this end, we used a rational in silico screening approach based on the known 


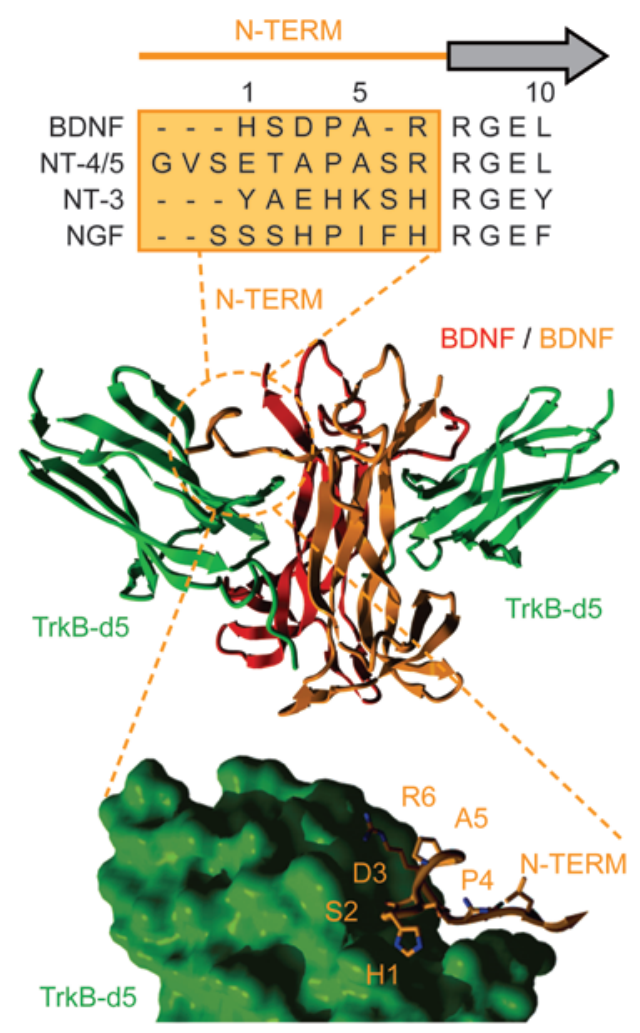

structural interaction between neurotrophins and Trk receptors. Using a similar in silico screening strategy but based on a BDNFderived pharmacophore approach, Massa and colleagues recently presented a small molecule that can activate TrkB and thereby prevent neurodegeneration in rats (24). A random screening strategy also recently led to the identification of a nonpeptide TrkB agonist with neurotrophic activities (25). In the present study, we identified ANA-12, which we believe is the first small molecule that antagonizes TrkB and that possesses potent anxiolytic-like and antidepressant-like activities in mice.

\section{Results}

Modeling of the BDNF/TrkB specificity patch and docking-based virtual screening. Previous studies have shown that the $\mathrm{N}$-terminal region of neurotrophins is critical for the binding selectivity and activation of their cognate Trk receptors (for review, see refs. 26, 27). Moreover, the crystal structure of NT-4/5 in complex with the fifth subdomain of TrkB (TrkB-d5) shows that the N-terminal region of the neurotrophin interacts with the "specificity patch" of the receptor, a binding pocket located in TrkB-d5 that is thought to drive the selectivity of the interaction with BDNF (Figure 1). Since no structural data are available for the BDNF/TrkB complex, we derived a $3 \mathrm{D}$ model from the existing high-resolution $\mathrm{x}$-ray structures of the NT-4/5/TrkB complex (28) and the BDNF/NT-3 dimer (29) (see Methods). The virtual screening strategy was performed using this model and by focusing on the specificity interaction patch between the BDNF N-terminal region and the TrkB-d5 domain (Figure 1). The Bioinfo-DB database of 1.6 million commercially available drug-like compounds (30) was first filtered for lead-like properties (31), including notably predicted water solubility and blood-brain barrier permeation, to afford 78,045

\section{Figure 1}

Computational modeling of the specificity patch. The structure-based virtual screening was performed by targeting the specificity interaction patch between the N-terminal segment of BDNF (N-TERM, red/ orange) and the d5 subdomain of TrkB (TrkB-d5, green). An alignment of the N-terminal amino acid sequence of rodent BDNF, NT-4/5, NT-3, and NGF is shown in the upper panel (the specific region is boxed; dashes represent gaps). The 3D model of the complex between the TrkB-d5 domain (green solid surface) and the BDNF N-terminal part $\left(\mathrm{H}^{1} \mathrm{SDPAR}^{6}\right.$, orange sticks) is illustrated in the lower panel.

compounds. Preliminary docking studies of the BDNF terminal epitope $\left(\mathrm{H}^{1} \mathrm{SDPAR}{ }^{6}\right)$ with various docking algorithms indicated that the FlexX program (32) was particularly well suited for docking in the TrkB specificity site. All 78,045 compounds were directly docked with the FlexX v2.2 program into the specificity patch of the TrkB-d5 domain, yielding 295 virtual hits that were finally clustered by maximal common substructures using ClassPharmer (31). A representative compound (best FlexX score) of each of the 22 Class Pharmer classes described by at least 3 compounds was finally selected for purchase and biological evaluation.

In silico screening identifies TrkB antagonists. Out of the 22 virtual hits, 12 molecules were obtained from commercial sources and tested in the presence or absence of BDNF in KIRA-ELISA assays, a method that rapidly quantifies TrkB activation in 96-well plates (20). In a first set of assays performed on recombinant human TrkB expressed in an inducible cell line (TetOn-rhTrkB), 2 compounds (N-T04 and N-T19; Figure 2A) were found to fully inhibit BDNF-induced TrkB phosphorylation and were selected for further pharmacological characterizations.

KIRA-ELISA concentration-response assays performed on TetOnrhTrkB cells showed that both N-T04 and N-T19 fully inhibited BDNF-induced TrkB activation with similar potencies, N-T19 being slightly more potent $\left(\mathrm{IC}_{50}=508 \pm 85\right.$ and $192 \pm 31 \mu \mathrm{M}$, respectively; Figure 2B). Interestingly, N-T04 also decreased basal TrkB activity (defined as the signal eliminated by the nonselective tyrosine kinase inhibitor K252a in the absence of BDNF). Using the same inducible TetOn-rhTrkB system, we previously demonstrated that this basal activity is dependent on TrkB density and can be inhibited by both K252a and cyclotraxin-B, a TrkB-specific allosteric modulator (20). Because of the high range of concentrations ( 0.1 to $1 \mathrm{mM}$ ) for which this effect occurs, we further verified that the decrease in TrkB activity was not due to toxic side effects. First, KIRA-ELISA assays showed that the amount of total TrkB proteins was unchanged when the level of phospho-TrkB was reduced after treatment with N-T04 alone. Second, morphological visualization of the cells did not indicate any toxic effects of N-T04 on TetOnrhTrkB cells. Together, these data suggest that N-T04, like K252a and cyclotraxin-B (20), inhibits both BDNF-dependent and -independent activity of TrkB receptors.

Since the purpose of this study was to develop agents that could act on TrkB in the brain, we then examined the effects of the 2 molecules on neurons. Cortical neurons naturally express TrkB together with $\mathrm{p} 75^{\mathrm{NTR}}$, a coreceptor that alters TrkB conformation and provides greater selectivity for BDNF (33). Surprisingly, N-T04 lost its ability to inhibit TrkB activity in neurons (Figure 2C). In contrast, N-T19 showed both a higher potency and a 2-site mechanism of action. Eadie-Hofstee plotting of the data suggested that the low-affinity site $(300 \mu \mathrm{M})$ resembles the site of action in TrkB only-expressing cells whereas the high-affinity site $(5 \mu \mathrm{M})$ 
A<smiles>Cc1cc(=O)oc2cc(NC(=O)[C@H](CCCC[NH3+])NC(=O)C(CC(C)C)NC(=O)C(C)[NH3+])ccc12</smiles><smiles>N#[W]c1cccs1</smiles>
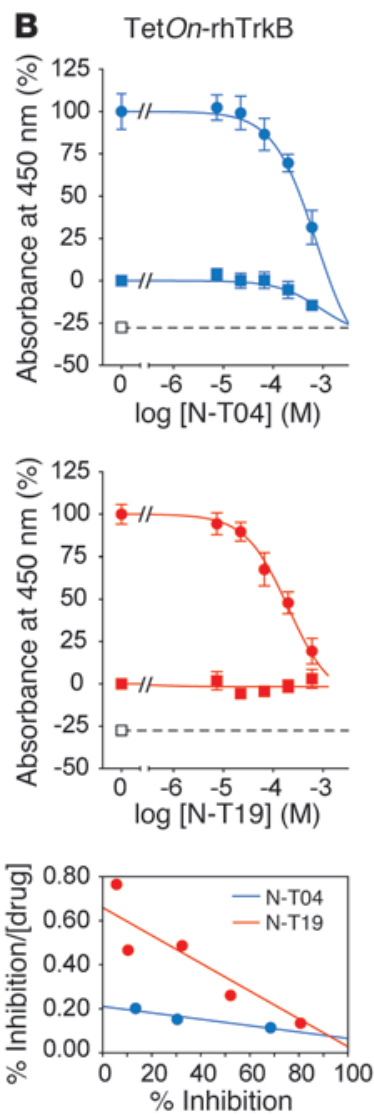
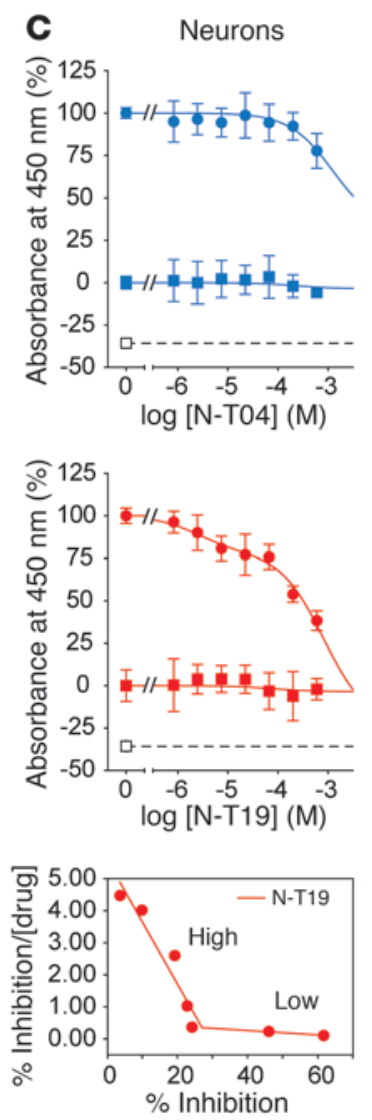
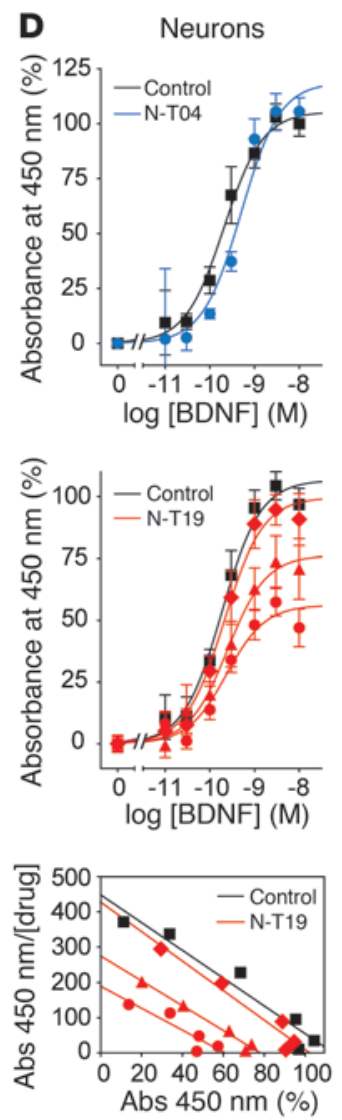

Figure 2

Pharmacological properties of N-T04 and N-T19 in different systems. (A) Structures of the 2 most active compounds, N-T04 and N-T19. (B and C) KIRA-ELISA assessment of N-T04 and N-T19 in absence (filled squares) or presence (filled circles) of BDNF in TetOn-rhTrkB and neuronal cells. Values were normalized to signal obtained with $1 \mathrm{nM}(\mathbf{B})$ or $0.4 \mathrm{nM}(\mathbf{C})$ of BDNF after subtraction of signal obtained in control wells. Compounds were compared with $10 \mu \mathrm{M}$ of K252a (open squares), a concentration that abolishes basal TrkB activity $(20)$. I $\mathrm{C}_{50}$ values of both high-affinity $(4.7 \pm 1.9 \mu \mathrm{M})$ and low-affinity $(231 \pm 53 \mu \mathrm{M})$ sites for N-T19 in neurons were determined using Eadie-Hofstee plotting of the data (lower panels). Nonlinear regression curve fit for N-T19 in neurons was obtained using a 2-site competition model. Data represent mean \pm SEM of 3-6 experiments performed in triplicate. (D) BDNF concentration-response curve in absence (squares) or in presence (diamonds, $3 \mu \mathrm{M}$; triangles, $30 \mu \mathrm{M}$; circles, $300 \mu \mathrm{M}$ ) of N-T04 or N-T19. A noncompetitive antagonism is clearly demonstrated for N-T19 by Eadie-Hofstee plotting of the data (lower panel). Values were normalized to the maximal BDNF response after subtraction of signal obtained in controls. Data represent mean \pm SEM of 4 experiments performed in triplicate.

was specific to neurons. This is reminiscent of the action of BDNF in neurons in which 2 binding sites, most likely due to the presence of $\mathrm{p} 75^{\mathrm{NTR}}$, are observed (33). To better characterize the pharmacological properties of the 2 compounds in neurons, BDNF concentration-response curves were performed in the absence or presence of N-T04 and N-T19 (Figure 2D). As expected, N-T04 did not affect the BDNF curve in neurons. In contrast, in the presence of increasing concentrations of N-T19, BDNF did not overcome N-T19 inhibition, suggesting a noncompetitive mechanism.

$N$-T19 inhibits cellular processes associated to TrkB. We then verified that both primary hits were able to modulate biological processes downstream of TrkB. BDNF is known to promote neurite outgrowth, notably in nnr5 PC12-TrkB cells, through a TrkB/MAPK signaling pathway activation. Addition of BDNF to the TrkB/ p $75^{\mathrm{NTR}}$-coexpressing nnr5 PC12-TrkB cells resulted in an increase in neurite length and number of branch points (Figure 3A). As previously observed in KIRA-ELISA assays, N-T04 did not produce any effect in these cells. However, N-T19 proved to dose depend ently prevent the BDNF-induced neurite outgrowth with the same pharmacological constants as those defined by KIRA-ELISA assays in cortical neurons (Figure 3B).

Screening of N-T19 analogs reveals a potent TrkB antagonist. KIRAELISA and neurite outgrowth assessments revealed that only N-T19 was able to maintain its effects in neurons and neuron-like systems. However, although N-T19 demonstrated a high efficacy in inhibiting TrkB activity, its relatively low potency prompted us to seek out analogs that keep the original high efficacy of N-T19 but with a better potency. For this purpose, a second round of in silico screening using the Bioinfo-DB database was undertaken to identify analogs of N-T19 sharing the same molecular scaffold (Figure 4A). Fourteen new molecules were identified as close analogs and were tested in a first set of functional screenings using KIRA-ELISA assays. Four compounds were found to have the highest activity but only 1 (ANA-12) demonstrated a submicromolar potency in recombinant cells. In-depth pharmacological characterizations of ANA-12 confirmed the high efficacy (full inhibition) 
A
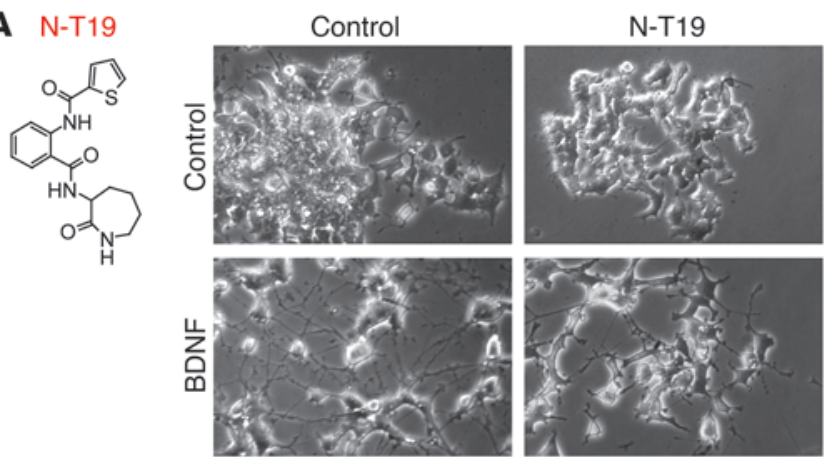

\section{B}
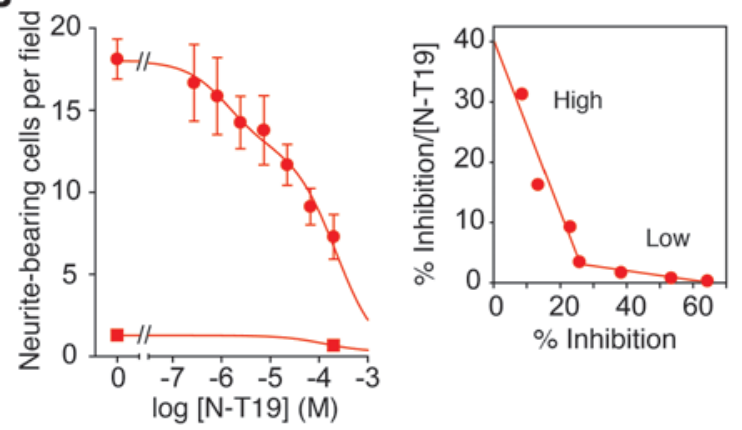

and potency (submicromolar) of the molecule (Figure 4B). Like the parent compound NT-19, ANA-12 revealed a 2-site mode of action in neurons but surprisingly also in recombinant cells. Potencies on the low- and high-affinity sites were comparable between the 2 cell systems (50 $\mu \mathrm{M}$ and $50 \mathrm{nM}$, respectively).

It is noteworthy that, of the 14 molecules tested, the structure of ANA-12 was the closest to that of the parent lead compound, both molecules differing only by an extra benzene moiety in ANA-12 (Figure 4A).

ANA-12 binds directly and selectively to TrkB. We then determined whether ANA-12 binds directly to TrkB by incubating the fluorescently labeled compound (see Methods) with chimeric TrkB ${ }^{\mathrm{ECD}}$-Fc or BSA or IgG-Fc as negative controls for nonspecific binding. As

\section{Figure 3}

N-T19 inhibits BDNF-induced neurite outgrowth. (A) Representative photomicrographs of nnr5 PC12-TrkB cells treated with N-T19 $(10 \mu \mathrm{M})$ and BDNF, alone or in combination as indicated. Original magnification, $\times 20$. (B) Quantitative analysis of control (squares) and BDNF-induced (circles) neurite outgrowth in presence of increasing concentrations of $\mathrm{N}$-T19. Nonlinear regression curve fit for was performed using a 2-site competition model. IC $\mathrm{C}_{50}$ values of both high-affinity $(1.5 \pm 0.2 \mu \mathrm{M})$ and low-affinity $(79 \pm 12 \mu \mathrm{M})$ sites were obtained by Eadie-Hofstee plotting of the data (inset, right panel). Data represent mean \pm SEM of 3 experiments performed six times each.

shown in Figure 5A, ANA-12 specifically bound to the extracellular domain of TrkB in a dose-dependent manner but not to BSA or IgG-Fc. Saturation binding studies with TrkB ${ }^{\text {ECD }}$-Fc showed that ANA-12 binds TrkB ECD $-F_{c}$ with a $K_{d}$ of $12 \mu \mathrm{M}$ (Figure $5 \mathrm{~B}$ ), which corresponds to the functional low-affinity site previously observed in KIRA-ELISA. The detection of the high-affinity site was located in the nonlinear range of fluorescence detection and therefore appeared as a small indentation. Linearization and extrapolation analyses showed that ANA-12 also binds the high-affinity site with a $K_{d}$ of approximately $10 \mathrm{nM}$ (data not shown). A 2-site fitting model of ANA-12 binding to TrkB demonstrated that the high-affinity site accounts for $20 \%$ of the total binding (data not shown), a value similar to the $30 \%$ observed in KIRA-ELISA assays (Figure 4B).

To further study the binding properties of ANA-12, BDNF was added to the ANA-12/TrkB complex (Figure 5B). This resulted in a $60 \%$ decrease in the maximal amount of ANA- 12 bound to TrkBECD_ Fc with no shift of the curve to the right $\left(K_{d}, 16 \mu \mathrm{M}\right)$, suggesting a noncompetitive mechanism. Together, these data suggest that both the high- and low-affinity binding sites coexist on the extracellular domain of TrkB and that BDNF and ANA-12 do not compete for the same sites on TrkB.

Computational docking of the new compound to TrkB-d5 demonstrated that the putative binding mode was similar to that of N-T19 (Figure 5C): the lactame moiety of ANA-12 interacts with the main chain atoms of His299 and His300 of TrkB, while hydrogen bonds are formed between the acyclic amide moiety of the compound and the side chains of TrkB-specific Gln347 and

\section{Figure 4}

Pharmacological properties of the N-T19 analog ANA-12. (A) Substructure query used to retrieve close analogs of $\mathrm{N}-\mathrm{T} 19$ (Ar, any aromatic moiety; R1 and R2, any moiety) and structure of ANA-12. The benzene moiety that differs from the template core is shown in green. (B) KIRA-ELISA assessment of ANA-12 in absence (filled squares) or in presence (filled circles) of BDNF in TetOn-rhTrkB cells and neurons, as described in Figure 2. $\mathrm{IC}_{50}$ values of the high-affinity (TetOnrhTrkB, $45.6 \pm 8.4 \mathrm{nM}$; neurons, $45.6 \pm 6.7 \mathrm{nM})$ and lowaffinity (TetOn-rhTrkB, $87.0 \pm 14.0 \mu \mathrm{M}$; neurons, $41.1 \pm 21.7$ $\mu \mathrm{M})$ sites were determined using Eadie-Hofstee plotting of the data (lower panels). Data represent mean \pm SEM of $2-4$ experiments performed in triplicates.
A

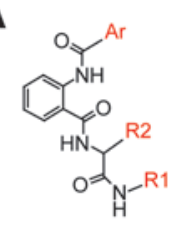

Template

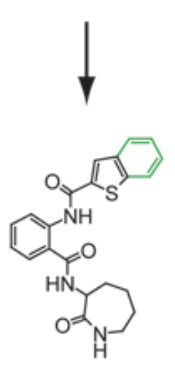

ANA-12
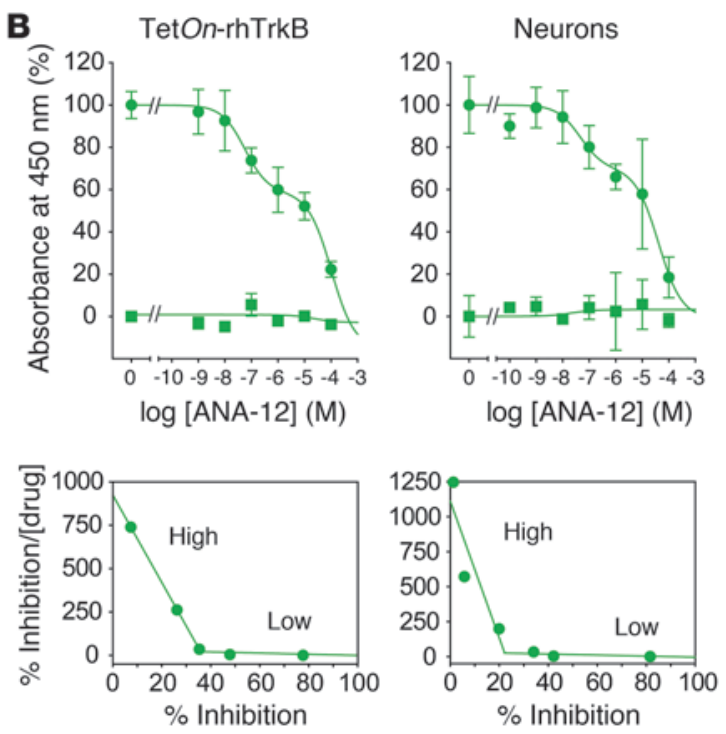

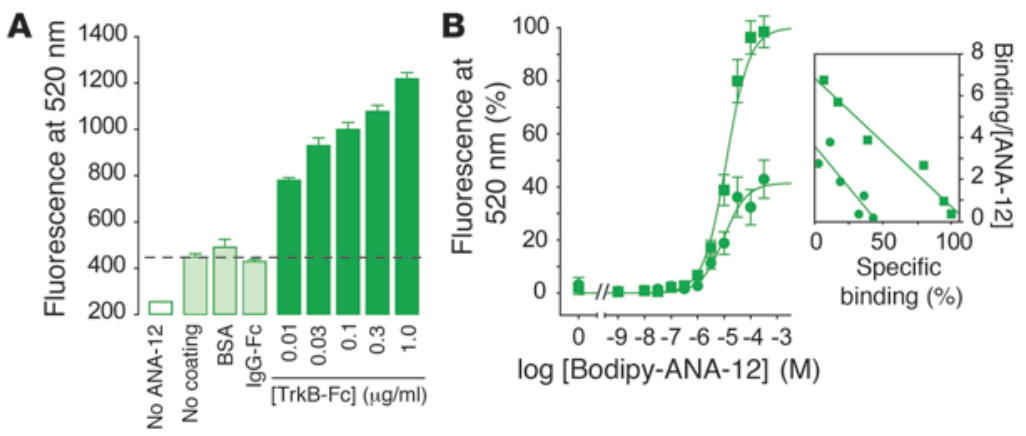

C

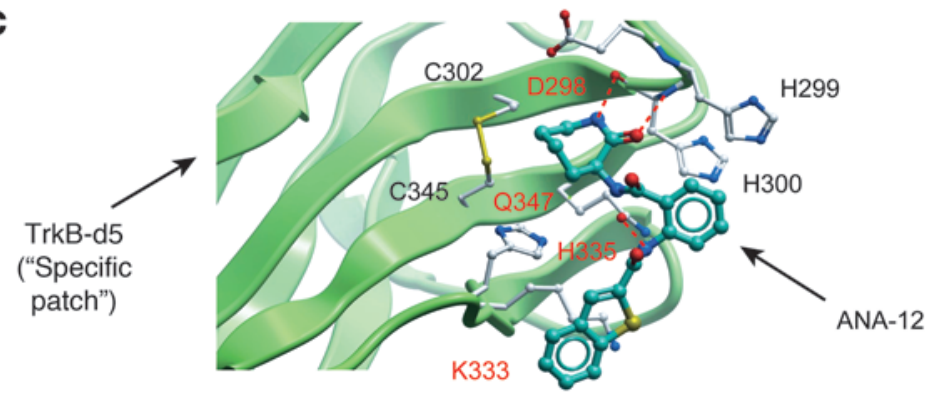

D

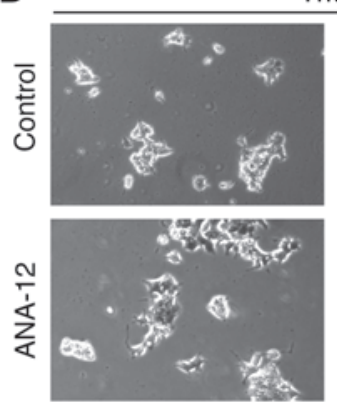

Control
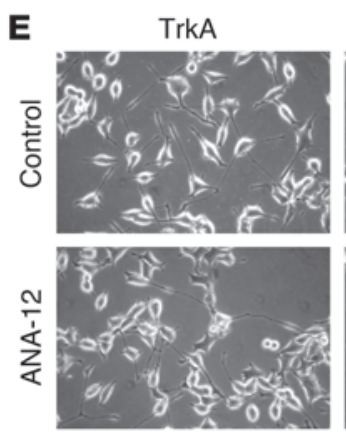

NGF
TrkB
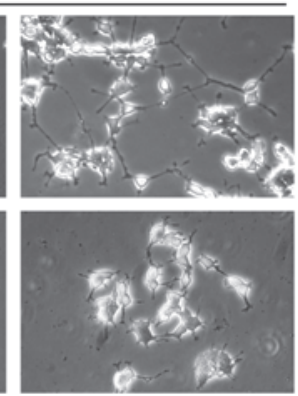

BDNF
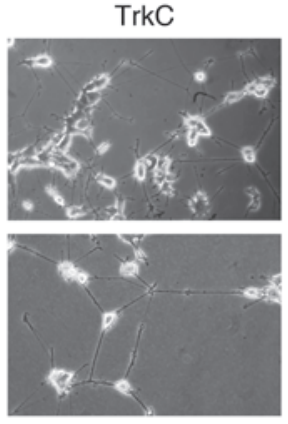

NT-3
TrkB
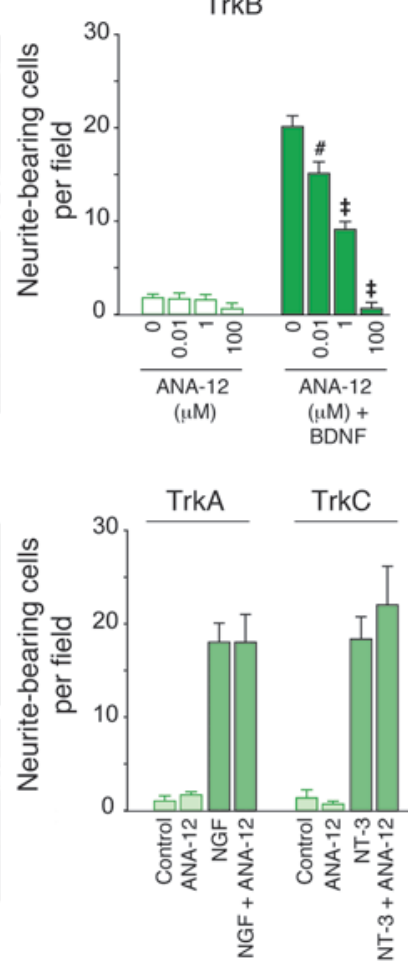

\section{Figure 5}

ANA-12 directly binds and selectively modulates TrkB. (A) Bodipy-ANA-12 (100 $\mu \mathrm{M})$ was incubated with increasing amounts of Trk ${ }^{B E C D}-F c$, IgG-Fc, BSA, or nothing (negative control; dashed line). Specific binding could be detected only with $\mathrm{Trk}^{\mathrm{BECD}}{ }^{\mathrm{F}} \mathrm{FC}$. Data represent mean \pm SEM of 2 experiments performed in triplicate. (B) Increasing concentrations of bodipy-ANA-12 were incubated with $\mathrm{Trk}^{\mathrm{BECD}}-\mathrm{Fc}(1 \mu \mathrm{g} / \mathrm{ml})$ in the presence (filled circles) or absence (filled squares) of 10 nM BDNF. Values are normalized to maximal signal after subtraction of nonspecific binding (BSA or IgG-Fc). Affinity constants $\left(K_{d}\right)$ were derived from Scatchard plotting of the data (inset, right panel). Data represent mean \pm SEM of 4 experiments performed in triplicate. (C) Computational model of the docking of ANA-12 (cyan) into the specificity patch of TrkB-d5 (green ribbon). 3 putative hydrogen bonds (red dotted lines) anchor ANA-12 to TrkB. Note that ANA-12 is surrounded by TrkB-specific amino acid residues (red) in the binding pocket. (D and $\mathbf{E})$ Representative photomicrographs and quantitative analyses of neurotrophin-induced neurite outgrowth in the presence of ANA-12 in nnr5 PC12-TrkB (D), -TrkA, and -TrkC (E) cells. Original magnification, $\times 20$. Data presented for TrkA and TrkC are those obtained with $100 \mu \mathrm{M}$ of ANA12 and are not different from their respective controls. ${ }^{\#} P<0.01$ and $¥ P<0.0001$. Data represent mean \pm SEM of 3 experiments performed in triplicate.
Asp298. The shape of the ligand fits into the TrkB-d5 ADEB $\beta$-sheet, notably through hydrophobic contacts between the 7-membered ring and the accessible disulfide bridge Cys302Cys347, and through aromatic-aromatic interactions with a bundle of histidine residues (His299, His300, His335).

ANA-12 affects cell functions associated with TrkB but not TrkA nor TrkC. Neurite outgrowth was used to verify the effects of ANA-12 on cellular processes (Figure 5, D and E). We observed that in the TrkB-expressing cells, ANA-12 prevented BDNF-induced neurite outgrowth at concentrations as low as $10 \mathrm{nM}$, confirming the high potency and efficacy observed in KIRA-ELISA assays (Figure 5D). At concentrations up to $10-100 \mu \mathrm{M}$, ANA-12 completely abolished the effects of BDNF since no single neurite or branching could be observed even after 3 days of incubation. To assess the selectivity of the compound toward TrkB, we used 2 other nnr5-PC12 cell lines expressing either TrkA or TrkC for which neurite outgrowth is dependent on NGF and NT-3, respectively (Figure 5E). In these cell lines, ANA-12 had no effect on neurite outgrowth. Three days 

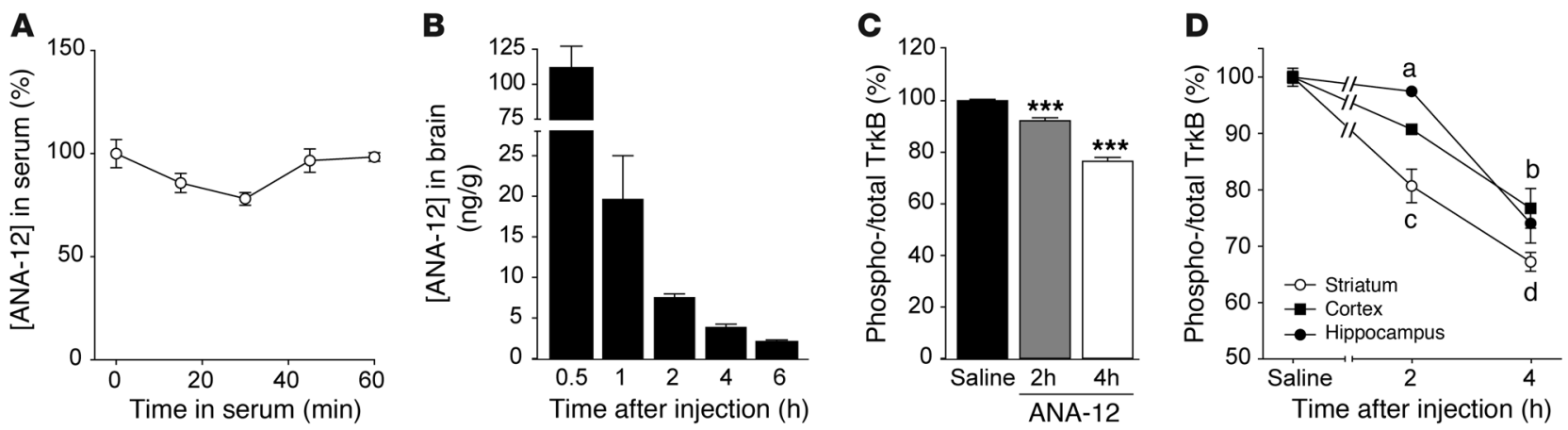

Figure 6

Injection of ANA-12 i.p. inhibits TrkB receptors in the brain. (A) ANA-12 stability in mouse serum. ANA-12 was incubated in serum of mice for 15 , 30,45 , and 60 minutes at $37^{\circ} \mathrm{C}$. Data represent mean \pm SEM of 2 experiments. (B) ANA-12 bioavailability in mouse brain, 30 minutes, $1,2,4$, and 6 hours after a single i.p. injection. Data represent mean \pm SEM of 3 animals/group. (C) Pharmacokinetics of TrkB inhibition in the brain following i.p. injection of saline or ANA-12 $(0.5 \mathrm{mg} / \mathrm{kg})$ into adult mice. The level of phospho- and total TrkB in the whole brain of saline-treated and ANA-12-treated mice was detected using KIRA-ELISA assays 2 and 4 hours after injection. Values are expressed as a percentage of the signal obtained for saline-treated animals for the 2 time points (data presented for the saline-treated group are those obtained at 2 hours but were similar at 4 hours). Data represent mean \pm SEM of 8 animals/group assessed in 2 different assays. ${ }^{* \star *} P<0.0001$ compared with saline. (D) Pharmacokinetics of TrkB inhibition in different brain regions. Data represent mean \pm SEM of 8 animals/group assessed in 2 different assays. Two-way ANOVA analyses of the data indicated a significant effect of treatment over time $(P<0.0001)$ and between structures $(P<0.0001)$. Post-hoc analysis showed significant effect in (a) striatum $(P<0.001)$ and cortex $(P<0.01)$ at 2 hours and in (b) all structures $(P<0.001)$ at 4 hours, compared with saline. At 2 hours (c), significant differences existed for striatum compared with cortex $(P<0.01)$ and hippocampus $(P<0.001)$, while at 4 hours (d), differences were significant between striatum and cortex $(P<0.01)$ and hippocampus $(P<0.05)$. For better clarity, the graph is magnified.

incubation with concentrations as high as $100 \mu \mathrm{M}$ of ANA-12 did not affect NGF- and NT-3-dependent neurite length and branching, confirming its specificity to TrkB-related signaling.

Systemic administration of ANA-12 inhibits TrkB in the brain. The purpose of this study was to develop a small molecule that can inhibit TrkB in the adult mammalian brain after systemic administration. We first tested whether ANA-12 was stable and not degraded into breakdown products in mouse serum before reaching the brain. To this end, ANA- 12 was incubated in mouse serum for $15,30,45$, and 60 minutes at $37^{\circ} \mathrm{C}$. Liquid chromatography-mass spectrometry (LC-MS) analysis of the mixture did not reveal the presence of breakdown products and no degradation of ANA-12 over time was observed (Figure 6A).

We then determined whether ANA-12 crosses the blood-brain barrier and reaches the brain after systemic administration. For this purpose, ANA-12 $(0.5 \mathrm{mg} / \mathrm{kg})$ was injected i.p. into adult mice. Animals were sacrificed $0.5,1,2,4$, or 6 hours later and brains were processed for quantification of ANA- 12 by LC-MS. Figure $6 \mathrm{~B}$ shows that active concentrations of ANA-12 could be detected in the brain as early as 30 minutes $(\sim 400 \mathrm{nM})$ and up to 6 hours after the i.p. injection $(\sim 10 \mathrm{nM})$.

We then determined whether ANA-12 inhibits TrkB in the adult brain and determined the magnitude of TrkB inhibition in the brain 2 and 4 hours after the injection of $0.5 \mathrm{mg} / \mathrm{kg}$ of ANA-12. This time course was chosen based on what we previously observed with cyclotraxin-B, for which a minimum of 3 hours was required for TrkB deactivation in the brain (20). KIRA-ELISA quantification of phospho-TrkB revealed that $0.5 \mathrm{mg} / \mathrm{kg}$ of ANA-12 partially inhibited the total endogenous TrkB activity in the whole brain ( $8 \%$ at 2 hours, $25 \%$ at 4 hours; Figure $6 \mathrm{C}$ ).

Although TrkB is widely distributed in the brain, it is possible that ANA-12 does not inhibit the receptor uniformly in different brain areas. This could lead to an apparent partial inhibition of TrkB in the whole brain with some structures being fully inhib- ited while others are not or are very little affected. We therefore determined the amplitude of inhibition between different brain areas. Again, $0.5 \mathrm{mg} / \mathrm{kg}$ of ANA-12 was injected into adult mice, and different brain structures (striatum, cortex, and hippocampus) were collected after 2 or 4 hours (Figure 6D). KIRA-ELISA analysis showed that $\operatorname{TrkB}$ inhibition was more effective in the striatum than in hippocampus and cortex 2 hours after injection. At 4 hours, inhibition was comparable among all the structures that were analyzed (25\%-30\%), yet TrkB in the striatum appeared to be slightly more inhibited than in the hippocampus and cortex. Together, these observations suggest that very low doses of ANA-12 are sufficient to partially inhibit TrkB activity homogenously throughout the brain after 4 hours.

Injection of ANA-12 into mice predicts anxiolytic and antidepressive activities. Overactivity of the BDNF/TrkB signaling in the reward circuitry, including striatum and cortex, and in the hippocampus has been associated with the development of anxiety and depression in rodent animal models $(7,13-15)$. We therefore tested the hypothesis that injecting ANA-12 into mice would predict efficient anxiolytic and antidepressant properties of the compound. To this end, adult mice received either $0.5 \mathrm{mg} / \mathrm{kg}$ of ANA- 12 or saline and were subjected to a panel of predictive tests that assess the anxiolytic and antidepressant potentials of a compound.

The elevated plus maze task is a standard exploratory test for the assessment of anxiety-related behaviors and is commonly used for its predictive validity for the screening of anxiolytic compounds (Figure 7A). As expected for anxiolytic compounds, mice treated with ANA-12 entered more often and spent more time in the aversive open arms while saline-treated mice preferentially stayed in the closed and protected arm.

To further assess the anxiolytic potential of ANA-12, saline- and ANA-12-treated mice were examined in the novelty-suppressed feeding paradigm. This test reflects anxiety- and depression-related behaviors because antidepressant and anxiolytic compounds 

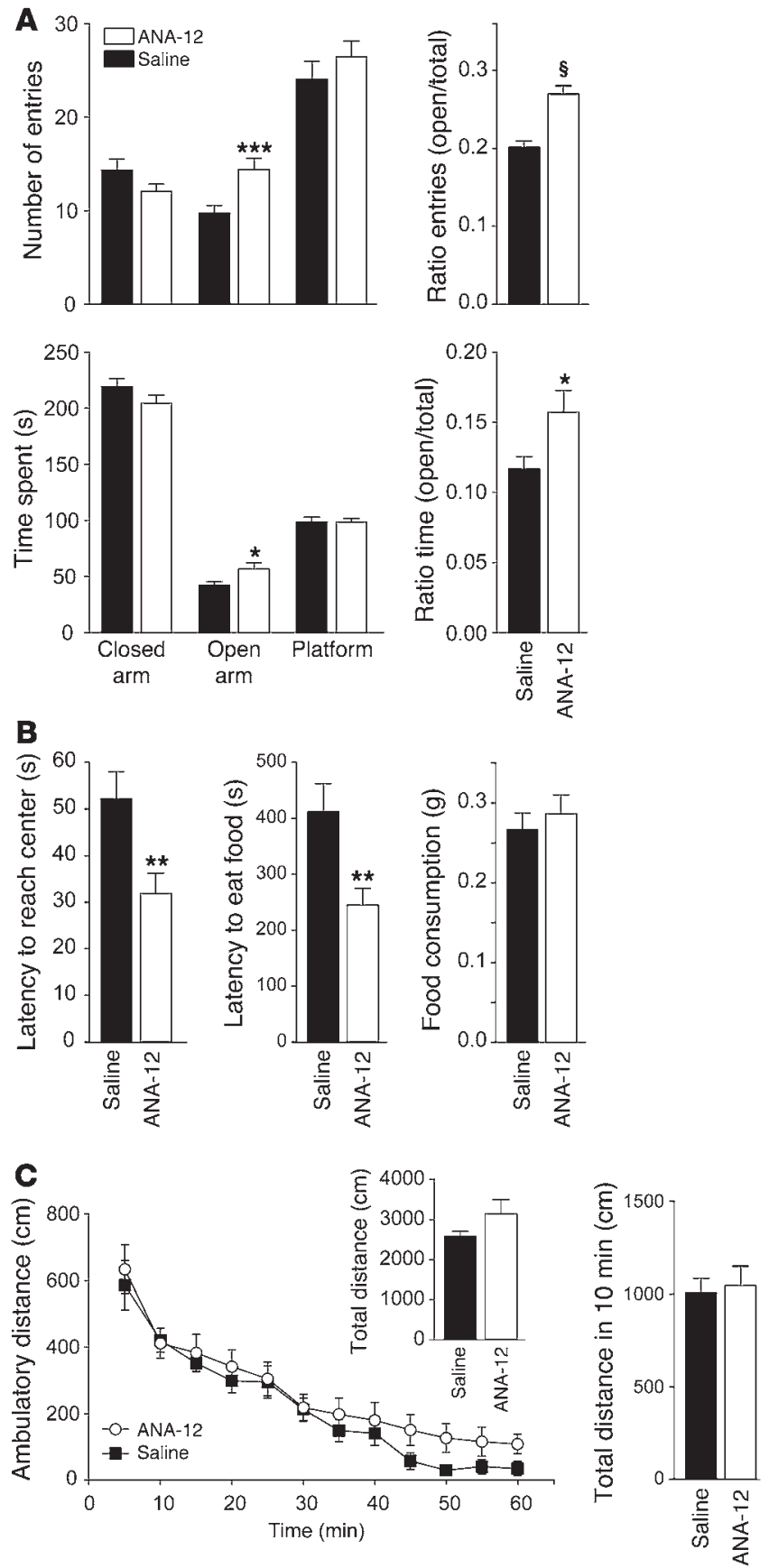

reduce the latency to reach the illuminated area and to begin feeding in an aversive environment (Figure 7B). In line with this, fooddeprived mice treated with ANA-12 entered the lit center and consumed the food pellet twice as fast as saline-treated mice. At the end of the test, food consumption in the home cage was assessed for 5 minutes to control the effect of the treatment on satiety. No difference was observed in the amount of food consumed by both groups, suggesting that the decreased latency was not due to differences in food deprivation.

General locomotor activity was not increased in the elevated plus maze and in the novelty-suppressed feeding task. To further rule out any confounding factors due to locomotor activity, we tested

\section{Figure 7}

ANA-12 demonstrates anxiolytic-like properties. (A) Exploratory behavior in the elevated plus maze. The number of entries and the time spent in the different arms of the maze are presented. The increased ratio of entries and time in the open arms versus total observed for ANA-12treated animals is representative of decreased anxiety. (B) Behavioral measures of saline-treated and ANA-12-treated mice in the novelty-suppressed feeding test. The latency to reach the illuminated center and to eat the food was dramatically decreased in animals that received ANA12 , while the home cage food consumption was not different between both groups. (C) Exploratory behavior in the novel open field of salinetreated and ANA-12-treated mice. Ambulatory distance was measured during 60 minutes and was not different between the 2 groups. Inset shows the total ambulatory distance acquired during the 60-minute session. The total distance covered during the first 10 minutes of the task is also shown. ${ }^{\star} P<0.05$; ${ }^{\star \star} P<0.01$; ${ }^{\star \star \star} P<0.005$; ${ }^{\S} P<0.001$, compared with saline. Data are presented as mean \pm SEM.

the exploratory behavior of vehicle- and ANA-12-treated mice in the open field under nonaversive light conditions (Figure 7C). The measure of ambulatory distance, used as an index of activity, was not different between saline-treated and ANA-12-treated animals, demonstrating that ANA-12 does not affect locomotor activity. Since all behavioral tasks consisted of a 10- to 15-minute session, we verified that locomotion was not affected by ANA- 12 within the first 10 minutes in the open field and we found that the total distance covered during this period was not different between the 2 groups.

To examine the potential antidepressant activity of ANA-12, we used 2 independent tasks, the Porsolt forced-swim test and the tail-suspension test. Both tests are commonly used for screening antidepressant properties of compounds because of their good reliability and predictive validity (34). Saline-treated and ANA-12treated mice were first subjected to the forced-swim test, and the duration of floating (immobility time) was measured (Figure 8A). In this task, mice treated with ANA-12 showed a decrease in the total floating time compared with saline-treated animals. In fact, saline-treated mice resigned after 4 minutes and until the end of the test, while ANA-12-treated animals did not show any increase in immobility during the whole test. This effect is a common feature of antidepressant compounds. Saline-treated and ANA-12treated mice were then subjected to the tail-suspension test and the duration of immobility was again measured. As expected for compounds with antidepressant properties, mice treated with ANA-12 were more resilient than saline-treated mice as they spent significantly less time immobile (Figure 8B).

ANA-12 does not affect neuron survival. Since inhibition of the BDNF/ TrkB signaling can induce neuronal death in the central nervous system $(2,35)$, we verified whether ANA-12 can be chronically administrated without toxic effects for the brain. For that purpose, adult mice received a daily injection of different doses of ANA-12 (0.5, 1.0, and $2.0 \mathrm{mg} / \mathrm{kg}$ ) or saline solution for a week. After the last injection, mice were sacrificed and brains were processed for apoptosis detection using fluorescent TUNEL staining (Figure 9). Little or no TUNELpositive cells could be detected in the brains of mice injected with saline or 0.5 or $1.0 \mathrm{mg} / \mathrm{kg}$ of ANA-12. It is noteworthy that examination of the whole brain revealed that the apoptotic cells were present only in the dentate gyrus of the hippocampus. However, a small increase in the number of TUNEL-positive cells was observed in the dentate gyrus of mice that received $2.0 \mathrm{mg} / \mathrm{kg}$ of ANA-12. No staining was detected in any other areas of investigation (cortex, striatum, 

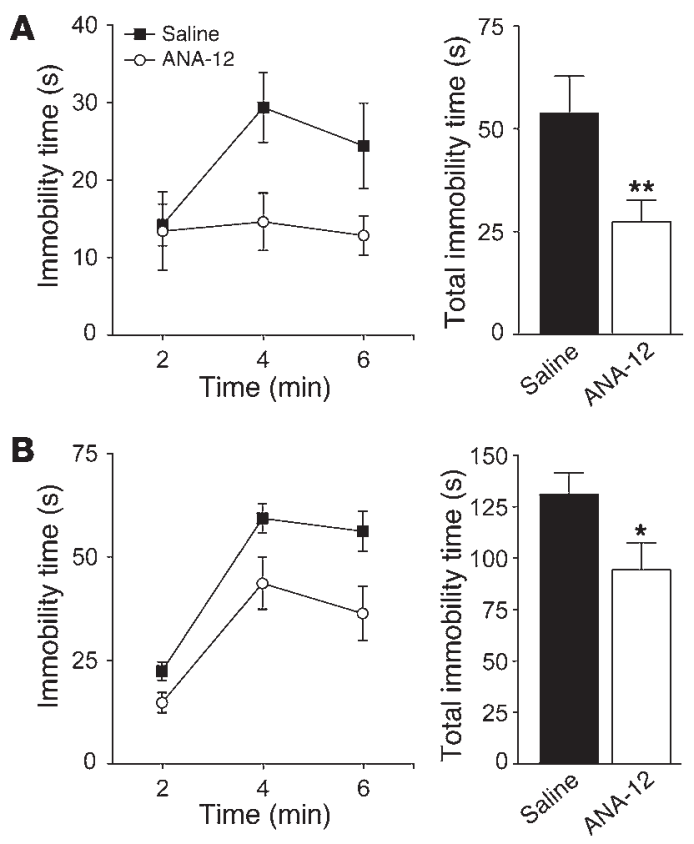

CA1-3 areas of the hippocampus, hypothalamus, thalamus, substantia nigra, ventral tegmental area, pallidum, and raphe nucleus).

\section{Discussion}

In this study, we identified what we believe is the first low-molecular weight antagonist of TrkB using a structure-based virtual approach. The identified molecule binds the extracellular domain of TrkB, prevents BDNF-induced TrkB activation, and abolishes the biological effects of BDNF on TrkB-expressing cells but not those of NGF or NT-3 on TrkA- and TrkC-expressing cells. Moreover, the compound inhibits TrkB function in the brain after systemic administration and demonstrates anxiolytic and antidepressant potential.

The iterative process of rationale-based in silico screenings combined with functional assays illustrates the power of modeling specific structural interactions between 2 proteins for the rapid identification of functional modulators. Finding low-molecular weight compounds that selectively disturb protein-protein interactions is notably a difficult endeavor. As of today, only $1 \%$ of the whole human proteome has been successfully targeted with smallmolecule compounds (36). Moreover, most of the fruitful reports concern proteins that naturally have small organic molecules for ligands and not another protein. The 2-digit nanomolar affinity observed for ANA-12 is therefore particularly encouraging for other protein-protein interfaces considering the pace and small resources engaged here to discover the current lead.

In order to allow rapid and efficient selection of functionally active ligands, we used a discrete and specific area of the TrkB

\section{Figure 9}

The behaviorally effective dose does not affect cell survival. Representative TUNEL staining in the dentate gyrus of mice subchronically injected with saline or ANA-12 $(0.5,1.0$, or $2.0 \mathrm{mg} / \mathrm{kg})$ are shown (Original magnification, $(\times 10)$. Positive (DNAse-treated sections) and negative (absence of terminal transferase) controls are included for comparison. Arrows show TUNEL-positive cells as microscopically determined under higher magnification $(\times 40)$. Scale bar: $100 \mathrm{~mm}$.

\section{Figure 8}

ANA-12 demonstrates antidepressant-like properties. (A) Saline- and ANA-12-treated animals were subjected to the forced-swim test. While saline-treated mice resigned after 4 minutes, mice that received ANA-12 were as active as at the beginning of the test. Two-way ANOVA analysis of the data demonstrated a significant overall effect of the treatment $(P<0.01)$. (B) This escape behavior was confirmed in the tail-suspension test, as demonstrated by the decrease in immobility $(P<0.001,2$-way ANOVA). Bar graphs show the total immobility time. ${ }^{\star} P<0.05 ;{ }^{\star \star} P=0.01$. Data are presented as mean \pm SEM.

receptor for the virtual docking of a large database of 1.6 million structurally unrelated compounds. This region is located in the fifth subdomain of the extracellular domain of TrkB and has been described as the "specificity patch" of the receptor, as it interacts with the $\mathrm{N}$-terminal region of $\operatorname{BDNF}(26,28)$. In fact, the $\mathrm{N}$-terminal end of neurotrophins has been shown (a) to be highly variable in terms of amino acid composition, (b) to affect Trk receptor binding and activation capacities, and (c) to fit into a binding pocket formed by a patch of charged amino acids in the fifth subdomain of Trk receptors. We therefore hypothesized that modeling the TrkB specificity patch to perform an in silico screening would lead to active TrkB-selective ligands. Conventional high-throughput screenings using functional assays, such as neurite outgrowth and cell survival, are demanding and generally yield less than $1 \%$ active compounds (37). Moreover, the identified active compounds are likely to act through any mechanism and not necessarily through TrkB receptors. In contrast, the structure-based virtual strategies used in this study obtained a high rate of recovery, with 2 active molecules out of 12 tested in the first round of screening and 4 out of 14 in the second round of screening for more potent analogs. Very recently, Massa and colleagues have discovered active TrkB agonists using a pharmacophore-based approach by modeling the variable and specific loop II in BDNF (24). This strategy yielded 5 active compounds out of 7 tested that can activate TrkB both in vitro and in vivo. Together, these findings demonstrate the effi-
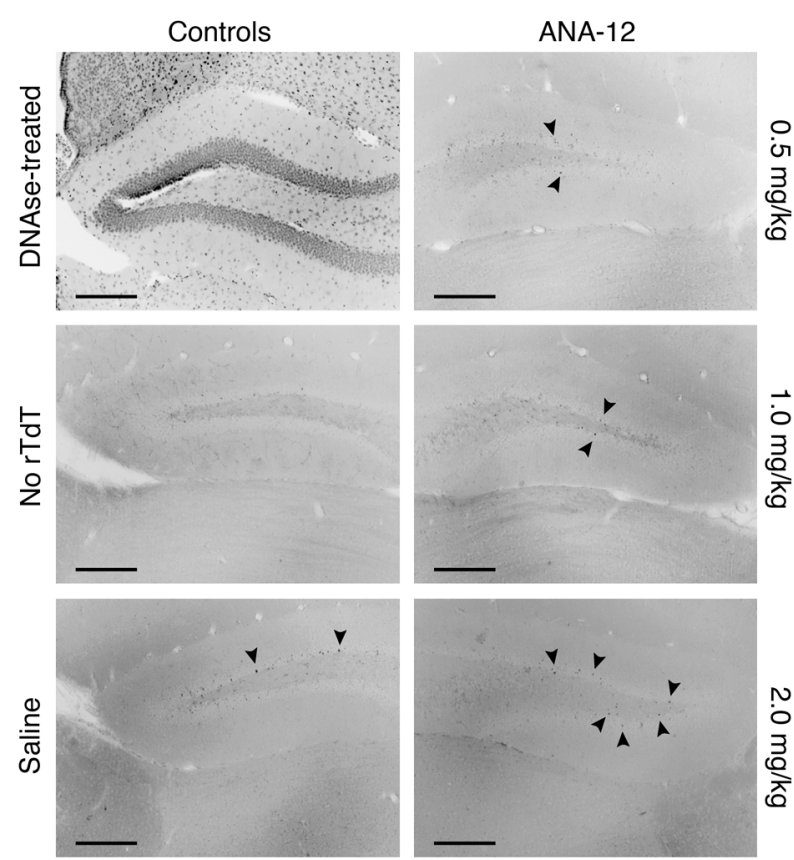
ciency of structure-based virtual screenings for the discovery of functional and selective Trk receptor ligands.

The data presented here show that low-molecular weight heterocyclic compounds affect the formation of a functional complex between BDNF and TrkB. Two of the compounds described here, ANA-12 and N-T19, structurally differ only by a benzene moiety, yet this modification showed important consequences on the pharmacological properties of ANA-12. For instance, while N-T19 demonstrated a second higher-affinity site when tested in neurons, this site was present in both recombinant and native systems for ANA-12. Here, we can hypothesize that N-T19 needs p $75^{\mathrm{NTR}}$ to modulate the conformation of TrkB-d5 in order to open a binding pocket that will facilitate its entry and result in an apparent higher potency. The addition of a benzene cycle to ANA-12 may overcome the need of $\mathrm{p} 75^{\mathrm{NTR}}$ to access this binding pocket. This would result in an apparent higher-affinity site even in the absence of $\mathrm{p} 75^{\mathrm{NTR}}$. Binding studies using TrkB ${ }^{E C D}$-Fc showed that ANA-12 selectively binds to the extracellular domain of TrkB. Interestingly, 2 binding sites could be detected with constants similar to those observed in functional assays (high-affinity site, $K_{d} \approx 10 \mathrm{nM}$, fraction $20 \%-30 \%$; low-affinity site, $K_{d} \approx 10 \mu \mathrm{M}$, fraction $70 \%-80 \%$ ). The presence of 2 binding sites on purified TrkB confirms the idea that ANA-12 may access a binding pocket independently of $\mathrm{p} 75^{\mathrm{NTR}}$-induced transconformation of TrkB.

Modeling of ANA-12 docking in the specificity patch showed that the molecule nicely fits into the TrkB-d5 ADEB $\beta$-sheet and behaves like the $\mathrm{N}$ terminus region of BDNF in the binding pocket $(26,28)$. Similarly to NT-4/5 and BDNF, ANA-12 lies against the disulfide bridge formed by Cys302 and Cys347 and interacts with Asp298, His299, and His300. Cocrystallization of the compound with TrkB, in the presence or absence of BDNF, would be of invaluable help for a better understanding of the fine molecular events triggering TrkB activation. In fact, binding experiments showed that even high concentrations of ANA-12 cannot overcome its displacement from TrkB by BDNF, suggesting a noncompetitive mechanism. Given the size of BDNF relative to ANA-12 and its many binding sites on TrkB (26-28), one can hypothesize that while the N-terminal arm of the neurotrophin competes with ANA-12 for the TrkB-d5-binding pocket, the small compound is not able to destabilize the other sites of interactions between BDNF and TrkB.

Systemic administration of low doses of ANA-12 to mice efficiently inhibited TrkB activity in the striatum, cortex, and hippocampus, and showed predictive anxiolytic and antidepressant properties in a panel of behavioral tests that included the elevated plus maze, the novelty-suppressed feeding, the tail suspension, and the Porsolt swimming tests. These tests are commonly used for their predictive validity to assess anxiolytic-like and antidepressant-like activities of compounds (38). Because BDNF has been described as a modulator of memory formation, the behavioral alterations observed in these tests could therefore theoretically be a result of a memory deficit. However, it has been shown that memory can be addressed in the elevated plus maze only when repeated trials and preexposure sessions are used (38-40), which is not the case here. Similarly, the novelty-suppressed feeding test measures the latency to first approach, which by definition cannot be affected by memory. We therefore exclude potential memory impairments as a confounding factor in our behavioral analysis.

The neurotrophin hypothesis of depression postulates that BDNF is strongly linked to depression and depression-like behaviors $(9,10)$. However, while positive modulation of BDNF sig- naling may be a key target for antidepressant actions, inhibition of this pathway does not seem to induce depression (16-18). In contrast, inhibition of BDNF and TrkB signaling in the reward system has shown strong antidepressant effects in rodent models of stress and depression $(7,12)$. Given the dynamic activity of the $\mathrm{BDNF} / \mathrm{TrkB}$ coupling in these disorders, it has been proposed that BDNF antagonists would provide a novel class of efficient therapeutic agents to treat anxiety and depression in humans $(7,11-15)$. Accordingly, we propose that ANA-12, antagonist of BDNF/TrkB signaling, may be effective for the treatment of anxiety disorders and depression in humans.

We previously observed similar behavioral effects on the elevated plus maze using cyclotraxin-B, a BDNF-derived peptide that inhibits both BDNF-dependent and -independent TrkB activity in the brain (20). Interestingly, cyclotraxin-B had no effect in the forced-swim test. Further investigations will be required to better understand the different mechanisms underlying the effects of these 2 compounds and more generally to better understand the role of BDNF and TrkB in mood disorders. A potential issue with the use of TrkB inhibitors for clinical application as anxiolytic and/or antidepressive medication could be the induction of cell death. Chronic treatments with such compounds could result in the loss of neurons, the survival of which depends on BDNF signaling (2). However, as demonstrated in this study with ANA-12, this side effect can potentially be prevented by using low doses of the antagonist that will efficiently alter behavior without affecting the survival of neurons.

Finally, other compounds sharing the 3-(acylamino)-e-caprolactam scaffold of N-T19 and ANA-12 have been described as procognitive agents in mice (41), although the precise mechanism of action is not described (42). Given the central role of the BDNF/ TrkB coupling in cognition and memory, it would be interesting to evaluate the effect of these compounds on TrkB receptors and to compare them with ANA-12 and N-T19.

To the best of our knowledge, ANA-12 is the first nonpeptide antagonist of TrkB receptor that elicits strong and specific effects in vivo. This proteolytically stable small molecule constitutes a valuable pharmacological tool to enable us to better investigate the role of BDNF/TrkB signaling in pathophysiological situations and will serve as a lead compound for the design of potent orally bioavailable TrkB modulators.

\section{Methods}

\section{Materials}

D-Ala-Leu-Lys-7-amido-4-methylcoumarin (N-T04; cat. no. A8171; M.W. $487.6 \mathrm{~g} / \mathrm{mol}$ ) was purchased from Sigma-Aldrich, with purity higher than $95 \%$ determined by HPLC. N2-(2-\{[(2-oxoazepan-3-yl)amino $]$ carbonyl $\}$ phenyl)thio phene-2-carboxamide (N-T19; (cat. no. BTB06524; M.W.357.4 g/mol) and N2(2-\{[(2-oxoazepan-3-yl)amino]carbonyl $\}$ phenyl)benzo[b]thiophene-2-carboxamide (ANA-12; cat. no. BTB06525; MW $407.5 \mathrm{~g} / \mathrm{mol}$ ) were purchased from Maybridge, with purity higher than $95 \%$ determined by LC-MS. Recombinant human BDNF, NGF, and NT-3 were obtained from Peprotech. TrkB ${ }^{\text {ECD }}$-Fc was obtained from R\&D Systems. Antibodies and substrates used for KIRAELISA were obtained from different sources, as follows: anti-GFP polyclonal antibody (BD Biosciences), anti-GFP monoclonal antibody (Chemicon), antiTrkB polyclonal antibody (Millipore), anti-TrkB monoclonal antibody (BD Biosciences), biotinylated anti-phosphotyrosine antibody (4G10; Upstate Biotechnology), HRP-conjugated streptavidin (Amersham Biosciences), and 3,3', 5',5-tetramethyl-benzidine substrate (TMB; Sigma-Aldrich). K252a was purchased from Calbiochem and doxycycline from Clontech. 


\section{Synthesis of Bodipy-ANA-12}

Bodipy-ANA-12 (2-(3-((2-(2-(2-(4-(benzo[b]thiophene-2-carboxamido)3-((2-oxoazepan-3-yl)carbamoyl)phenoxy)ethoxy)ethoxy)ethyl)amino)3-oxopropyl)-5,5-difluoro-7,9-dimethyl-5H-dipyrrodol[1,2-c:2', $\left.1^{\prime}-f\right]$ $[1,3,2]$ diazaborinin-4-ium-5-uide) was synthesized as described in Supplemental Methods.

\section{D modeling of the BDNF/TrkB complex}

Atomic coordinates of the BDNF-TrkB complex (2 TrkB molecules complexed to a BDNF homodimer) were obtained by homology to the $\mathrm{x}$-ray structure of the TrkB/NT-4/5 complex (28) (RCSB Protein Data Bank entry, 1 hcf). NT- $4 / 5$ coordinates were replaced by those of the BDNF $x$-ray structure (RCSB Protein Data Bank entry, 1 bnd) after fitting BDNF to NT-4/5 structures on $C \alpha$-atoms from residues Gly8 to Lys116. Missing coordinates for the $\mathrm{N}$-terminal part of BDNF (His1-Arg7) were obtained from that of the NT- $4 / 5$ template in the TrkB/NT- $4 / 5$ complex by substituting NT-4/5 residues with BDNF residues using the SYBYL Biopolymer package (Tripos Associates Inc.). A residue deletion when comparing NT- $4 / 5$ to BDNF (occurring at Ser9, NT- $4 / 5$ numbering) was generated by directly linking Ala6 to Arg7 BDNF residues from the NT-4/5 backbone template. After adding hydrogen atoms using standard SYBYL geometries, the BDNF-TrkB complex was refined in AMBER 8.0 using the ff03 forcefield (43) by 5,000 steps of energy minimization (1,000 steps of steepest descent and 4,000 steps of conjugate gradients refinement) with an implicit generalized Born solvation model.

\section{Docking-based virtual screening of the BDNF/TrkB specificity patch}

TrkB residues closer than $6.5 \AA$ to any of the $\mathrm{N}$-terminal BDNF amino acids (His1-Ser11) were selected for docking a database of commerciallyavailable drug-like compounds (Bioinfo database, http://bioinfo-pharma. u-strasbg.fr/bioinfo). 3D coordinates of compounds were obtained from available 2D sketches using Corina v3.1 (Molecular Networks GmbH) ionized at physiological $\mathrm{pH}$ with filter v2.0 (OpenEye Scientific Software) and saved in mol2 format. Out of 1.6 million compounds, an automated workflow was set-up in Pipeline Pilot v7.5 (Accelrys Inc.) to select 78,045 commercially available molecules to be screened using the following criteria: (a) lead-like properties of the compounds, as described earlier (31); (b) a predicted solubility in aqueous solution at $100 \mathrm{mM}$; and (c) a polar surface area below $120 \AA^{2}$. In silico screening was performed using the FlexX v2.2 docking software (32) using standard parameters. Any compounds with a FlexX score above -25.0 were selected for visual inspection. Any pose for which the ligand was buried by more than $75 \%$ of its surface upon TrkB docking was retrieved. All 295 selected ligands were clustered based on their maximal common substructures in ClassPharmer v4.5 (Simulation Plus Inc.). 22 ligands (N-T01 to N-T22) described by at least 3 compounds were selected. Twelve compounds were retrieved from commercial sources and dissolved in DMSO.

\section{Screening of N-T19 analogs}

The chemical scaffold of N-T19 (N-\{2-[(carbamoylmethyl)carbamoyl] phenyl arylamide) was used as a template for the screening of the Bioinfo database. 14 analogs (ANA-01 to ANA-14) were selected, retrieved from commercial suppliers, and dissolved in DMSO.

\section{Cell cultures}

Generation and selection of the inducible TetOn-rhTrkB cells expressing the recombinant human TrkB (provided by Garrett Brodeur, Children's Hospital of Philadelphia, Philadelphia, Pennsylvania, USA) tagged with the enhanced cyan fluorescent protein (rhTrkB-ECFP) were described earlier
(20). TetOn-rhTrkB cells were grown in DMEM (Invitrogen) supplemented with $10 \%$ bovine serum, $25 \mathrm{mM}$ Hepes (Invitrogen), $2 \mathrm{mM}$ 1-glutamax (Gibco), and the appropriate drug selection. Cortical neurons were prepared from E16 mouse embryos and cultured as previously described (20). The nnr5 PC12-TrkA, nnr5 PC12-TrkB, and nnr5 PC12-TrkC cells (provided by Moses Chao, Skirball Institute of Biomolecular Medicine, New York, New York, USA) are NGF nonresponding mutant PC12 cells stably transfected with TrkA, TrkB, and TrkC cDNA, respectively (44).

\section{KIRA-ELISA}

TrkB receptor autophosphorylation was quantified using a modified version of KIRA-ELISA, as described earlier (20). Tet $O n$-rhTrkB cells were seeded on flat-bottom 96-well culture plates $\left(4 \times 10^{4}\right.$ cells per well $)$ and were incubated overnight with $1000 \mathrm{ng} / \mathrm{ml}$ doxycycline to induce the expression of TrkB receptors. Cortical neurons were seeded on polyornithin-coated flat-bottom 96 -well culture plates $\left(12 \times 10^{4}\right.$ cells per well $)$ and cultured 7 to 8 days at $37^{\circ} \mathrm{C}$ in $5 \% \mathrm{CO}_{2}$. Fluorescence levels in Tet $O n$-rhTrkB cells were verified before each assay. Cells were carefully washed 4 times with DMEM before being treated with compounds for 20 minutes and stimulated for 20 minutes with BDNF (recombinant cells, $4 \mathrm{nM}$; neurons, $0.4 \mathrm{nM}$ ) in DMEM containing $0.5 \% \mathrm{BSA}$ and $25 \mathrm{mM}$ HEPES (control medium) at $37^{\circ} \mathrm{C}$ in $5 \%$ $\mathrm{CO}_{2}$. Assay was stopped by removing the medium on ice, and membranes were solubilized by adding a solubilization buffer $(150 \mathrm{mM} \mathrm{NaCl}, 50 \mathrm{mM}$ Hepes, $0.5 \%$ Triton X-100, $0.01 \%$ thimerosal, $2 \mathrm{mM}$ sodium orthovanadate, supplemented with a cocktail of protease inhibitor) for 1 hour at room temperature. Lysates were transferred to an ELISA microtiter plate (Nunc MaxiSorp) precoated with either anti-GFP (1:5000) for rhTrkB or anti-TrkB $(1 \mu \mathrm{g} / \mathrm{ml})$ for neuronal TrkB. Phosphorylation was revealed by incubation with biotinylated anti-phosphotyrosine $(0.5 \mu \mathrm{g} / \mathrm{ml})$ and HRP-conjugated streptavidin (1:4000). After addition of TMB and acidification with $1 \mathrm{~N}$ hydrochloric acid, absorbance was read at $450 \mathrm{~nm}$. Total TrkB was also quantified using KIRA-ELISA using either a monoclonal anti-GFP (1:3000) for rhTrkB or a monoclonal anti-TrkB (1:1000) for neuronal TrkB to precipitate the receptor and either a polyclonal anti-GFP (1:5000) for rhTrkB or a polyclonal anti-TrkB $(1 \mu \mathrm{g} / \mathrm{ml})$ for neuronal $\operatorname{TrkB}$ to detect the receptor. In cell cultures, the total TrkB signal was found to be unchanged in all treatment conditions. In brain tissues, the amount of total TrkB was variable and was used to normalize the signal obtained for phospho-TrkB.

\section{ANA-12 binding assays}

Maxisorp ELISA 96-well plates were coated with various concentrations of $\operatorname{Trk}^{\text {BECD }}-\mathrm{Fc}$ (as indicated in the figures), $20 \mathrm{mg} / \mathrm{ml} \mathrm{BSA}$, or $1 \mathrm{mg} / \mathrm{ml} \mathrm{IgG}-\mathrm{Fc}$ (polyclonal anti-TrkB; Millipore) in a carbonate buffer ( $\mathrm{pH}$ 9.6) overnight at $4{ }^{\circ} \mathrm{C}$. Plates were saturated with $0.5 \%$ BSA in PBS for 2 hours at room temperature and extensively washed in PBS-Tween 0.05\%. Bodipy-ANA-12 was then incubated in $0.5 \%$ PBS-BSA for 1 hour at room temperature before the addition of BDNF in $0.5 \%$ PBS-BSA for another hour, as indicated in the figures. After extensive washes in PBS-Tween $0.05 \%$, the amount of bodipyANA-12 bound was quantified by fluorescence at $520 \pm 10 \mathrm{~nm}$. Detectability range for extrapolation analysis was assessed by coating ELISA plates with bodipy-ANA-12 and reading fluorescence at $520 \pm 10 \mathrm{~nm}$.

\section{Neurite outgrowth assay}

Modulation of neurite outgrowth by molecules was assessed in nnr5 PC12-TrkB, -TrkA, and -TrkC cells after addition of BDNF (1 nM), NGF ( $2 \mathrm{nM})$, and NT-3 (10 nM), respectively. The number of cells bearing neurites longer than 2 cells in diameter in each counting field was microscopically determined (2 fields per well, 3 wells per condition). Counting was performed blind each 24 hours for 3 days (data presented in figures are those obtained for 48 hours but are similar at 24 and 72 hours). 


\section{Institutional review of animal protocols}

All animal protocols used in the present study were approved by the Institutional Animal Care and Use Committee of Columbia University and the INSERM Committee Guidelines and European Communities Council Directive.

\section{Administration of ANA-12 and in vivo KIRA-ELISA analysis}

F1 hybrids obtained by crossing C57BL/ 6 and $129 \mathrm{SveV}$ mice were used following the recommendation of the Banbury Conference on genetic background for studying mouse behavior (45). Adult C57BL6/129SveV F1s (3 months old) were randomly distributed into saline (1\% DMSO dissolved in $0.9 \% \mathrm{NaCl}$ solution) and ANA-12 (dissolved in saline solution) groups. A volume of $10 \mu \mathrm{l} / \mathrm{g}$ body weight was injected i.p. for saline and ANA-12 ( $0.5 \mathrm{mg} / \mathrm{kg}$ body weight) solutions. After 2 or 4 hours, mice were decapitated and brains were rapidly removed on ice. Striatum, cortex, and hippocampus were subsequently dissected when needed. Tissues were rapidly washed in ice-cold PBS, transferred into ice-cold KIRA-ELISA solubilization buffer, and left overnight at $4^{\circ} \mathrm{C}$. Protein concentrations were determined, equal amounts of proteins were loaded, and KIRA-ELISA assays were performed as described above.

\section{Analysis of ANA-12 stability and bioavailability in mouse brains \\ Analysis of stability in mouse serum and quantification in mouse brain were performed by TechMed ${ }^{\mathrm{ILL}}$ facilities (École Supérieure de Biotechnolo- gie de Strasbourg, Illkirch, France). Stability was assessed by incubating ANA-12 in mouse serum for $15,30,45$, and 60 minutes at $37^{\circ} \mathrm{C}$. Mixtures were homogenized and proteins precipitated with acetonitrile before being analyzed by liquid chromatography-mass spectrometry (Agilent LC-MS ESI qTOF connected to a C18-1 × $10 \times 1.9$ column). Bioavailability in mouse brain was assessed by injecting mice i.p. with ANA-12 $(0.5 \mathrm{mg} / \mathrm{kg})$ as described above. After 30 minutes, 1, 2, 4, and 6 hours ( 3 mice/time point), brains were removed and mashed in $\mathrm{NaCl} 0.9 \%$ before treatment with ace- tonitrile. The mixture was cleared by ultracentrifugation, and the com- pound was extracted using a series of dehydrations and resuspensions in acetonitrile/water $(1 / 1 \mathrm{v} / \mathrm{v})$ before being subjected to LC-MS for detection and analysis. Reference samples were prepared by adding known amounts of compound to blank mixtures (brains from saline-treated mice). Concen- trations of ANA-12 (ng/g brain) were derived from the reference samples by calculating the area under the detection peak.}

\section{In vivo cell death analysis}

The effects of subchronic treatments of ANA-12 on cell death in mouse brains were assessed using the TUNEL assay. Mice were randomly distributed into 4 groups and were i.p. injected with saline (containing $1 \%$ DMSO) or $0.5,1.0$, or $2.0 \mathrm{mg} / \mathrm{kg}$ of ANA-12 once a day for 1 week. Mice were sacrificed 24 hours after the last injection and perfused transcardially with PBS followed by $4 \%$ paraformaldehyde. Brains were postfixed overnight in $4 \%$ paraformaldehyde, and $50 \mu \mathrm{m}$-thick coronal sections were obtained using a vibratome. Free-floating sections were extensively washed in PBS and permeabilized in PBS containing 0.5\% Triton X-100 for 90 minutes at room temperature. Sections were then rinsed and mounted on slides. Labeling of 3' OH-DNA strand breaks was performed using the DeadEnd Fluorometric TUNEL system (Promega) according to the manufacturer's instruction. The reaction was terminated by rinsing the slides in SSC $2 \times$ and washing extensively with PBS. Sections were mounted with VECTASHIELD plus DAPI (Vector Laboratories), and fluorescein was visualized at $520 \mathrm{~nm}$ using fluorescence microscopy (Leica). Positive controls were obtained by pretreating sections from saline-treated animals with DNAse $(10 \mathrm{U} / \mathrm{ml}$; Invitrogen) for 90 minutes at $37^{\circ} \mathrm{C}$ in PBS containing $0.5 \%$ Triton $\mathrm{X}-100$. Negative controls (absence of fluorescent spots) were obtained by processing the sections as described above without the terminal transferase.

\section{Behavioral testing of ANA-12}

All behavioral testing was started at the same time of the day (1:00 pm) in quiet and separated rooms under bright ambient light conditions (800-900 lux, except for the open field, 300-400 lux). Behavioral tasks were performed 4 hours after injection of $0.5 \mathrm{mg} / \mathrm{kg}$ of ANA-12 using the same procedure as described above. To eliminate odor cues, all testing apparatus was thoroughly cleaned after each animal using the disinfectant Roccal (Pfizer).

\section{Effects of ANA-12 on anxiety-related behaviors}

Anxiety-related behaviors were tested using the open field test, elevated plus maze, and novelty-suppressed feeding paradigm.

Open field. The open field test was performed as previously described (46). Briefly, mice were tested in Plexiglas activity chambers $(45 \times 45 \times 30 \mathrm{~cm}$, $l \times w \times h$, model ENV-520; Med Associates) equipped with infrared beams located $1.5 \mathrm{~cm}$ above the chamber floor and spaced $2.5 \mathrm{~cm}$ apart to detect horizontal activity. Mice were placed into the center of the open field, and activity was recorded for 60 minutes. The center area was defined as the central $15 \times 15 \mathrm{~cm}$ portion. Total ambulatory distance, total ambulatory time in the periphery, and total ambulatory time in the center were measured.

Elevated plus maze. The elevated plus maze task is a standard exploratory test for the assessment of anxiety-related behavior and was performed as previously described (46). Mice were placed on the center platform facing a closed arm and allowed to explore the maze for 5 minutes. During these 5 minutes of testing, the number of entries and time spent in each arm were recorded.

Novelty-suppressed feeding. The novelty-suppressed feeding test captures both depression- and anxiety-related behaviors and is sensitive to antidepressants and anxiolytics (47). The test was performed with 24-hour fooddeprived mice as previously described (46). The testing apparatus consisted of a plastic box $(50 \times 50 \times 20 \mathrm{~cm}, l \times w \times h)$. The floor was covered with wood chip bedding. A single food pellet was placed on a circular piece of white filter paper (12 cm diameter) placed in the illuminated center of the box. Mice were placed in a corner of the box and the latency to reach the pellet and begin a feeding episode was recorded (maximum time 12 minutes). Each mouse was weighed prior to food deprivation and testing. To control for the effect of drug treatment on performance, each mouse was transferred to its home cage immediately after the test and the amount of food consumed in 5 minutes was measured.

\section{Effects of ANA-12 on depression-related behaviors}

Depression-related behaviors were tested using the forced-swim test and the tail-suspension test.

Forced-swim test. The forced-swim test was performed according to the method of Porsolt (48). A vertical glass cylinder $(25 \times 14 \mathrm{~cm}, h \times d)$ was filled with $30^{\circ} \mathrm{C}$ water to a depth of $20 \mathrm{~cm}$ so that mice must swim or float without their hind limbs or tail touching the bottom. For testing, each mouse was placed in the cylinder for 6 minutes, and duration of floating (immobility time) was scored. As indicated by Porsolt, only the data scored during the last 4 minutes were pooled and analyzed as total immobility time.

Tail-suspension test. The tail-suspension test was performed as described by Steru et al. (49). A short piece of paper adhesive tape was attached along half the length of the tail (about $3 \mathrm{~cm}$ ). The free end of the adhesive tape was attached to a $30-\mathrm{cm}$ long rigid tape attached to a frame so that the mouse's head was about $20 \mathrm{~cm}$ above the floor. The animal was surrounded by a white-painted wooden enclosure $(40 \times 40 \times 45 \mathrm{~cm}, l \times w \times b)$. Mice were observed for 6 minutes. Resignation to the aversive situation (immobility time) was recorded.

\section{Statistics}

Concentration-response and competition curves were obtained using an iterative least-squares method derived from that of Parker and Waud (50). 
Eadie-Hofstee plotting of the data provided estimates for $\mathrm{EC}_{50}$ and $\mathrm{IC}_{50}$ values. KIRA-ELISA competition studies were analyzed using 1-way ANOVA followed by Neuman-Keuls multiple comparison post hoc test. Concentration-response curves and Eadie-Hofstee plots were analyzed using 2-way ANOVA. In vivo KIRA-ELISA statistical analyses were performed using 1-way and 2-way ANOVA followed by Dunnett's multiple comparison and Bonferroni's post hoc tests. Behavioral data are presented as mean \pm SEM of 15 animals/group and were analyzed using either 2-tailed Student's $t$ test, 1-way ANOVA, or 2-way ANOVA with repeated measures where appropriate. $P<0.05$ was considered significant.

\section{Acknowledgments}

We are grateful to P. Facchinetti for cortical neuron cultures and J. Fuss for helping with the behavioral tests. The authors would like to thank G. Brodeur for the gift of the full human TrkB sequence and M. Chao for providing the nnr5 PC12 cells.

Received for publication June 10, 2010, and accepted in revised form February 23, 2011.

Address correspondence to: Maxime Cazorla, Columbia University, Department of Psychiatry, Physicians and Surgeons Building, Room 7-431, 630 West 168th Street, New York, New York 10032, USA. Phone: 212.342.3115; Fax: 212.305.5594; E-mail: mc3195@ columbia.edu. Or to: Didier Rognan, Structural Chemogenomics, Laboratory of Therapeutic Innovation, UMR-7200 CNRS/Université de Strasbourg, 74 route du Rhin, Illkirch, F-67400, France. Phone: 33.3.68.85.42.35; Fax: 33.3.68.85.42.10; E-mail: rognan@unistra.fr.
1. Yano H, Chao MV. Neurotrophin receptor structure and interactions. Pharm Acta Helv. 2000; 74(2-3):253-260.

2. Kaplan DR, Miller FD. Neurotrophin signal transduction in the nervous system. Curr Opin Neurobiol. 2000;10(3):381-391.

3. Bramham CR, Messaoudi E. BDNF function in adult synaptic plasticity: the synaptic consolidation hypothesis. Prog Neurobiol. 2005;76(2):99-125.

4. Lu Y, Christian K, Lu B. BDNF: a key regulator for protein synthesis-dependent LTP and long-term memory? Neurobiol Learn Mem. 2008;89(3):312-323.

5. Graham DL, Edwards S, Bachtell RK, DiLeone RJ, Rios M, Self DW. Dynamic BDNF activity in nucleus accumbens with cocaine use increases self-administration and relapse. Nat Neurosci. 2007;10(8):1029-1037.

6. Chen ZY, et al. Genetic variant BDNF (Val66Met) polymorphism alters anxiety-related behavior. Science. 2006;314(5796):140-143.

7. Berton O, et al. Essential role of BDNF in the mesolimbic dopamine pathway in social defeat stress. Science. 2006;311(5762):864-868.

8. Castren E, Rantamaki T. The role of BDNF and its receptors in depression and antidepressant drug action: Reactivation of developmental plasticity. Dev Neurobiol. 2010;70(5):289-297.

9. Duman RS, Monteggia LM. A neurotrophic model for stress-related mood disorders. Biol Psychiatry. 2006; 59(12):1116-1127.

10. Wang JW, Dranovsky A, Hen R. The when and where of BDNF and the antidepressant response. Biol Psychiatry. 2008;63(7):640-641.

11. Martinowich K, Manji H, Lu B. New insights into BDNF function in depression and anxiety. Nat Neurosci. 2007;10(9):1089-1093.

12. Eisch AJ, et al. Brain-derived neurotrophic factor in the ventral midbrain-nucleus accumbens pathway: a role in depression. Biol Psychiatry. 2003;54(10):994-1005.

13. Krishnan V, et al. Molecular adaptations underlying susceptibility and resistance to social defeat in brain reward regions. Cell. 2007;131(2):391-404.

14. Pardon MC, et al. Social threat and novel cage stress-induced sustained extracellular-regulated kinase1/2 (ERK1/2) phosphorylation but differential modulation of brain-derived neurotrophic factor (BDNF) expression in the hippocampus of NMRI mice. Neuroscience. 2005;132(3):561-574.

15. Fanous S, Hammer RP Jr, Nikulina EM. Short- and long-term effects of intermittent social defeat stress on brain-derived neurotrophic factor expression in mesocorticolimbic brain regions. Neuroscience. 2010; 167(3):598-607.

16. MacQueen GM, et al. Performance of heterozygous brain-derived neurotrophic factor knockout mice on behavioral analogues of anxiety, nociception, and depression. Behav Neurosci. 2001;115(5):1145-1153.

17. Saarelainen T, et al. Activation of the TrkB neurotrophin receptor is induced by antidepressant drugs and is required for antidepressant-induced behavioral effects. J Neurosci. 2003;23(1):349-357.

18. Zorner B, et al. Forebrain-specific trkB-receptor knockout mice: behaviorally more hyperactive than “depressive”. Biol Psychiatry. 2003;54(10):972-982.

19. Pu L, Liu QS, Poo MM. BDNF-dependent synaptic sensitization in midbrain dopamine neurons after cocaine withdrawal. Nat Neurosci. 2006;9(5):605-607.

20. Cazorla M, et al. Cyclotraxin-B, the first highly potent and selective TrkB inhibitor, has anxiolytic properties in mice. PLoS One. 2010;5(3):e9777.

21. Rashid K, et al. A nerve growth factor peptide retards seizure development and inhibits neuronal sprouting in a rat model of epilepsy. Proc Natl Acad SciUS A. 1995;92(21):9495-9499.

22. Colangelo AM, et al. A new nerve growth factormimetic peptide active on neuropathic pain in rats. J Neurosci. 2008;28(11):2698-2709.

23. Debeir T, Saragovi HU, Cuello AC. A nerve growth factor mimetic TrkA antagonist causes withdrawal of cortical cholinergic boutons in the adult rat. Proc Natl Acad Sci U S A. 1999;96(7):4067-4072.

24. Massa SM, et al. Small molecule BDNF mimetics activate $\operatorname{TrkB}$ signaling and prevent neuronal degeneration in rodents. J Clin Invest. 2010;120(5):1774-1785.

25. Jang SW, et al. A selective TrkB agonist with potent neurotrophic activities by 7,8-dihydroxyflavone. Proc Natl Acad Sci U S A. 2010;107(6):2687-2692.

26. Pattarawarapan M, Burgess K. Molecular basis of neurotrophin-receptor interactions. J Med Chem. 2003;46(25):5277-5291

27. Ibanez CF. Emerging themes in structural biology of neurotrophic factors. Trends Neurosci. 1998; 21(10):438-444.

28. Banfield MJ, Naylor RL, Robertson AG, Allen SJ, Dawbarn D, Brady RL. Specificity in Trk receptor: neurotrophin interactions: the crystal structure of TrkB-d5 in complex with neurotrophin-4/5. Structure. 2001;9(12):1191-1199.

29. Robinson RC, Radziejewski C, Stuart DI, Jones EY. Structure of the brain-derived neurotrophic factor/ neurotrophin 3 heterodimer. Biochemistry. 1995; 34(13):4139-4146.

30. Kellenberger E, Springael JY, Parmentier M, HachetHaas M, Galzi JL, Rognan D. Identification of nonpeptide CCR5 receptor agonists by structure-based virtual screening. JMed Chem. 2007;50(6):1294-1303.

31. Krier M, Bret G, Rognan D. Assessing the scaffold diversity of screening libraries. J Chem Inf Model. 2006;46(2):512-524.

32. Rarey M, Kramer B, Lengauer T, Klebe G. A fast flexible docking method using an incremental construction algorithm. J Mol Biol. 1996;261(3):470-489.

33. Chao MV, Hempstead BL. p75 and Trk: a two-receptor system. Trends Neurosci. 1995;18(7):321-326.

34. Cryan JF, Markou A, Lucki I. Assessing antidepressant activity in rodents: recent developments and future needs. Trends Pharmacol Sci. 2002;23(5):238-245.
35. Linnarsson S, Willson CA, Ernfors P. Cell death in regenerating populations of neurons in BDNF mutant mice. Brain Res Mol Brain Res. 2000;75(1):61-69.

36. Whitty A, Kumaravel G. Between a rock and a hard place? Nat Chem Biol. 2006;2(3):112-118.

37. Shoichet BK. Virtual screening of chemical libraries. Nature. 2004;432(7019):862-865.

38. File SE. The interplay of learning and anxiety in the elevated plus-maze. Behav Brain Res. 1993; 58(1-2):199-202.

39. Lister RG. The use of a plus-maze to measure anxiety in the mouse. Psychopharmacology (Berl). 1987; 92(2):180-185.

40. Deacon RM, Rawlins JN. T-maze alternation in the rodent. Nat Protoc. 2006;1(1):7-12.

41. Giannessi F, Ghirardi O, Misiti D, Tinti M, Cozzolino R, Scolastico C, inventors; Sigma-Tau Industrie Farmaceutiche Riunite S.p.A., assignee. 1-alkyl3-(acylamino)-epsilon-caprolactames as enhancers of learning and memory and pharmaceutical compositions containing same. US patent $5,155,102$. October 13, 1992.

42. Angelucci L, et al. Synthesis and amnesia-reversal activity of a series of 7- and 5-membered 3-acylamino lactams. J Med Chem. 1993;36(11):1511-1519.

43. Duan Y, et al. A point-charge force field for molecular mechanics simulations of proteins based on condensed-phase quantum mechanical calculations. J Comput Chem. 2003;24(16):1999-2012.

44. Green SH, Rydel RE, Connolly JL, Greene LA. PC12 cell mutants that possess low- but not high-affinity nerve growth factor receptors neither respond to nor internalize nerve growth factor. J Cell Biol. 1986; 102(3):830-843.

45. Silva AJ. Mutant mice and neuroscience: recommendations concerning genetic background. Banbury Conference on genetic background in mice. Neuron. 1997;19(4):755-759.

46. Ansorge MS, Zhou M, Lira A, Hen R, Gingrich JA. Early-life blockade of the 5-HT transporter alters emotional behavior in adult mice. Science. 2004; 306(5697):879-881.

47. Bodnoff SR, Suranyi-Cadotte B, Quirion R, Meaney MJ. A comparison of the effects of diazepam versus several typical and atypical anti-depressant drugs in an animal model of anxiety. Psychopharmacology (Berl). 1989;97(2):277-279.

48. Porsolt RD, Bertin A, Jalfre M. Behavioral despair in mice: a primary screening test for antidepressants. Arch Int Pharmacodyn Ther. 1977;229(2):327-336.

49. Steru L, Chermat R, Thierry B, Simon P. The tail suspension test: a new method for screening antidepressants in mice. Psychopharmacology (Berl). 1985; 85(3):367-370

50. Parker RB, Waud DR. Pharmacological estimation of drug-receptor dissociation constants. Statistical evaluation. I. Agonists J Pharmacol Exp Ther. 1971; 177(1):1-12. 